\title{
A series of Ti-Hf-Sn-Ta alloys produced in solid-state as prospective biomedical materials
}

K.O. Núñez-Acosta ${ }^{1}$, I. Estrada-Guel ${ }^{2}$, A. Torres-García ${ }^{3}$, M. Rocha-Rubio ${ }^{1}$, J.F. Hernández-Paz ${ }^{3}$, C.A. Rodríguez-González ${ }^{3}$ and C.D. Gómez-Esparza ${ }^{3}$

${ }^{1}$ Universidad Tecnológica de Ciudad Juárez, Ciudad Juarez, Chihuahua, Mexico, ${ }^{2}$ Centro de Investigación en Materiales Avanzados (CIMAV), Laboratorio Nacional de Nanotecnología, Miguel de Cervantes No. 120, CP 31136, Chihuahua, Chih., México., Chihuahua, Chihuahua, Mexico, ${ }^{3}$ Universidad Autónoma de Ciudad Juárez, Ciudad Juarez, Chihuahua, Mexico

In the last decades, a great advance in the field of biomaterials has been achieved. The Ti-based alloy implants have shown high biocompatibility, however, in sliding and friction functions, titanium suffers significant wear. The main cause of failures in medical implants is due to tribological causes. With the aim of extending the life cycle of prostheses, biomedical coatings have been developed to improve the biological and mechanical interaction of implant-bone through combine the properties of substrate and coating. On the other hand, recent investigations are focused on the development of Ti-based alloys exploring different alloying elements. Titanium has an HCP crystalline structure, called alpha $(\alpha)$ that can be transformed into a BCC structure, called beta $(\beta)$. B-type Ti alloys have recently been developed with the aim of improved the mechanical performance of the biomaterial. It has been reported that the addition of metallic elements such as hafnium (Hf), tin ( $\mathrm{Sn}$ ), and tantalum (Ta) favors the formation of the $\beta$ phase in Ti-based alloys [1]. Tantalum has excellent chemical stability, a high level of corrosion resistance, and excellent biocompatibility due to the formation of protective tantalum oxide. It is used in the manufacture of surgical instruments and implants. The Sn and $\mathrm{Hf}$ in Ti-based alloys decrease the elastic modulus improving the mechanical biocompatibility with human bone [1,2]. Under the concept of medium-entropy alloys [3], the aim of this investigation is the synthesis of a series of equiatomic Ti-Hf-Sn-Ta alloys by powder metallurgy, and evaluate the microstructural behavior, phase composition, and hardness as a function of chemical composition, and subsequently determine its potential use as a wear-resistant coating on Ti-based dental implants. A series of equiatomic Ti-Sn, Ti-Ta, TiSnTa, and TiHfSnTa alloys were synthesized by mechanical alloying (MA) and subsequent conventional sintering. Pure elemental powders with a high purity level $>99 \%$ were used as starting materials. The MA process was performed for $10 \mathrm{~h}$ under an inert Ar atmosphere. The ball-to-powder weight ratio was 10:1. The alloyed powders were compacted and sintered under vacuum at $1200^{\circ} \mathrm{C}$. The microhardness results of sintered samples were 987, 1023, 882, and $1074 \mathrm{HV}$ for the TiSn, TiTa, TiSnTa, and TiHfSnTa alloys, respectively. In the binary alloys, the Ta addition improves the hardness compared to Sn equiatomic content, while the combined effect of Sn and Ta in the ternary alloy produces a decrease between 10 and $15 \%$ in the hardness value. The quaternary alloy retains a hardness value similar to that of the Ti-Ta binary alloy. Fig. 1 shows the microstructure of sintered alloys. All of them exhibit a Ti-rich continuous phase with a rounded-like discontinuous phase, except for the TiSn alloy, the formation of tantalum oxide bright phase is observed. 


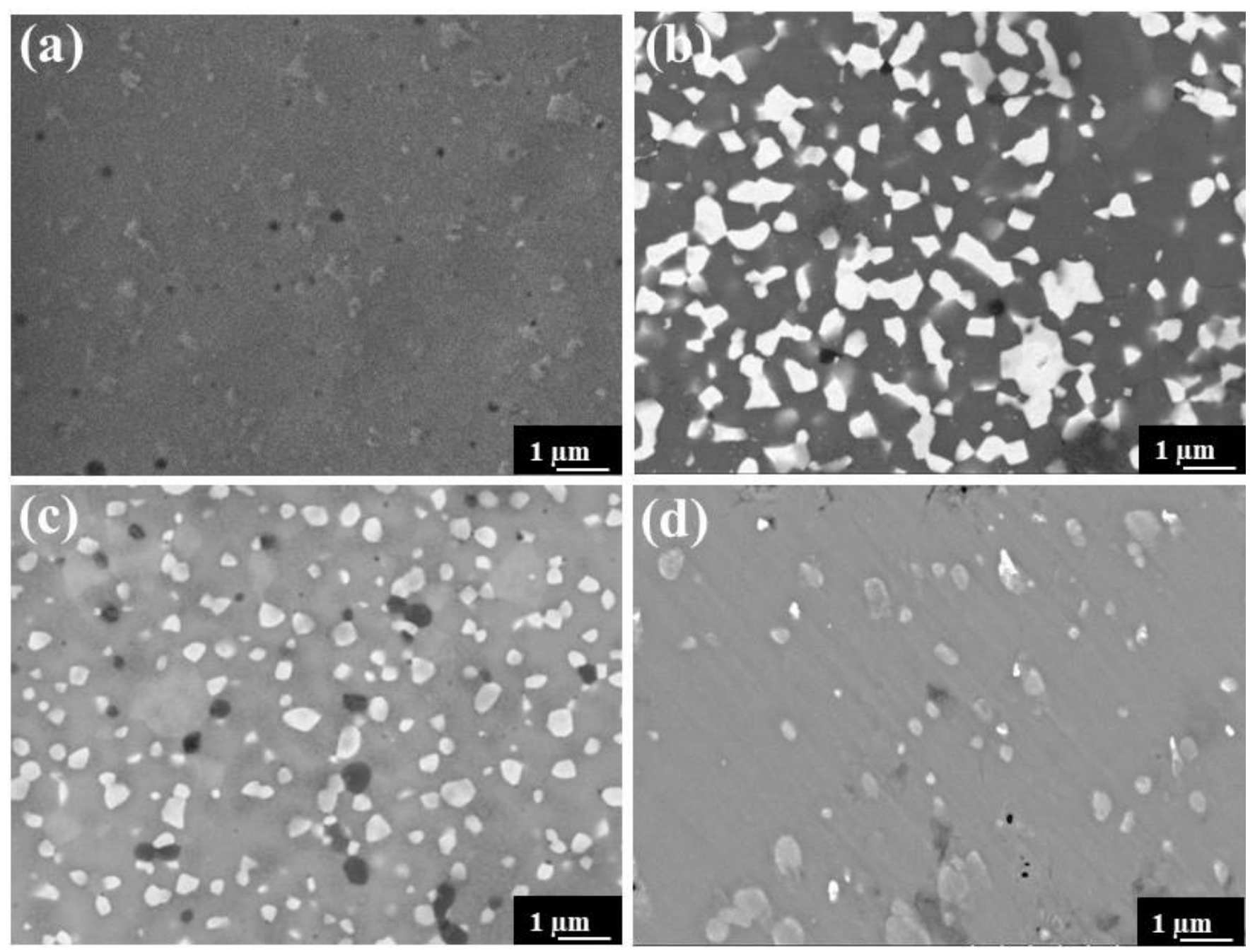

Figure 1. Figure 1. SEM-BSE micrographs of sintered alloys: a) TiSn, b) TiTa, c) TiSnTa, and d) TiHfSnTa.

References

[1] Y. Li, Ch. Yang, H. Zhao, S. Qu, X. Li and Y. Li, Materials 7 (2014) p. 1709.

[2] M. González, J. Peña, F.J. Gil, J.M. Manero, Mater. Sci. Eng. C 42 (2014) p. 691.

[3] Y. Zhou, D. Zhou, X. Jin, L. Zhang, X. Du, B. Li, Sci. Rep. 8 (2018). 